\title{
Influence of grain size on deuterium transport and retention in self-damaged tungsten
}

\author{
Matic Pečovnik ${ }^{1 *}$, Sabina Markelj ${ }^{1}$, Anže Založnik ${ }^{1}$ and Thomas \\ Schwarz-Selinger ${ }^{2}$ \\ ${ }^{1}$ Jozef Stefan Institute, Jamova cesta 39, 1000 Ljubljana, Slovenia \\ ${ }^{2}$ Max-Planck-Institut für Plasmaphysik, Boltzmannstrasse 2, D-85748 Garching, \\ Germany
}

\begin{abstract}
The influence of grain size on deuterium transport and retention in tungsten was studied. For this purpose an experiment was carried out on three polycrystalline tungsten samples with different grain sizes and a single crystal sample with surface orientation $<100>$. In order to increase deuterium retention and hence the sensitivity for detection, samples were first damaged by high energy $\mathrm{W}$ ions. After damaging the samples were exposed to a flux of deuterium atoms at $600 \mathrm{~K}$ for 70 hours. During the exposure the depth profile of the retained deuterium was measured by Nuclear Reaction Analysis using a ${ }^{3} \mathrm{He}$ ion beam. After the exposure the samples were also analysed by Thermal Desorption Spectroscopy. A clear difference in the time dependence of deuterium uptake was noticed between different samples. The experimental results were modeled using a rate-equation model. The influence of different grain size was modeled by changing the effective height of the potential barrier for deuterium atoms to enter into the bulk. We managed to successfully describe the transport of deuterium into the bulk of tungsten by reducing the potential barrier for samples with smaller grain sizes while the barrier for the sample with larger grain size was close to the value for the damaged single crystal sample.
\end{abstract}

Keywords: tungsten, deuterium transport, deuterium retention, grains, NRA PACS: 28.52.Fa, 07.77.-n, 25.55.-e, 68.43.-h, 66.30.je, 07.05.Tp, 61.72.Mm *Corresponding author: matic.pecovnik@ijs.si

\section{Introduction}

Tungsten is currently considered as one of the most promising materials for application in future fusion reactors, due to its low sputter yield, high thermal conductivity and very high melting temperature $(3700 \mathrm{~K})$. It is envisioned that the divertor target of the International Thermonuclear Experimental Reactor (ITER), where plasma is in direct contact with the material yielding the highest thermal and particles loads, will be built out of tungsten. One of the main issues regarding future use of fusion as an energy source which still needs to be resolved is the retention of radioactive tritium in the plasma-facing components. Tungsten has a very low hydrogen isotope solubility but the neutron induced damage which will reach up to a few displacements per atom (dpa) in DEMO will increase the amount of retained tritium in the tungsten divertor by orders of magnitude compared to undamaged tungsten [1]. Namely, the lattice defects created by $14 \mathrm{MeV}$ neutron irradiation act as trapping sites for hydrogen isotopes with high de-trapping energy as compared to the energy of diffusion between solution sites. The effect of defects in tungsten on hydrogen 
isotope retention is a well-researched topic [2, 3] but the role of parameters of the material, such as grain size, that may also have an effect on hydrogen isotope retention and transport, is still unclear. There is some theoretical work on hydrogen behaviour at grain boundaries, a recent review is given by Lu et al. [4. For example it has been shown that the $\Sigma 3(111)$ title grain boundary can trap up to 6 hydrogen atoms at low temperature which significantly weakens the cohesion of the material at the grain boundary. Multiple trapping of hydrogen isotopes (HI) in a grain boundary can also act as a precursor to HI bubble growth. By employing Molecular Dynamics simulations it was also shown that the activation energies for migration paths of HI into the grain boundary from the bulk are asymmetric and favor segregation of HI in the grain boundaries. It has also been shown that effective diffusion rates in grain boundaries are enhanced compared to diffusion through the bulk.

In the paper by Oda [5] a thermodynamic model was used to predict the effect of grains on hydrogen isotope effective solubility and effective diffusivity. By using his model to fit experimental data available in the literature he deduced that the effect of grain boundaries could be summarized as grain boundaries acting as a one dimensional defect with a binding energy $E_{\text {bind }}^{\text {grain }}=0.8-1.2 \mathrm{eV}$ and a modified diffusion barrier for hydrogen isotopes diffusing along the grain $E_{\text {diff }}^{\text {grain }}=0.3-0.5$ $\mathrm{eV}$. To the author's knowledge there exist only few experiments that deal with the effect of grain size [6] and nanostructure [7] on hydrogen isotope retention but none on the transport.

In this article the dependence of grain size on hydrogen isotope retention and transport in self-damaged tungsten is investigated experimentally and by modelling using a 1D Macroscopic Rate Equation model (MRE). To study the effects of grain size on deuterium transport in the bulk of the material we have used three samples with different grain sizes and one single crystal sample with surface orientation $<100>$. They have been damaged by high energy W ions (so-called self-damaging) creating lattice damage in the material and consequently increasing deuterium retention and sensitivity. After damaging, samples were exposed to low energy ( 0.27 $\mathrm{eV}$ ) deuterium atoms at $600 \mathrm{~K}$ for 70 hours, to populate the traps induced by the high energy tungsten ion implantation. During the exposure in situ measurement of the transport of deuterium into the bulk was performed using Nuclear Reaction Analysis (NRA) with a ${ }^{3} \mathrm{He}$ beam. After the exposure, Thermal Desorption Spectroscopy (TDS) was carried out. The experimental results were simulated using the TESSIM code [8], where we tried to simulate the differences between samples observed in the experiment by changing only the effective height of the potential barrier for deuterium migration from a surface adsorption site into the bulk.

\section{Model}

In order to describe the results of various experiments studying hydrogen isotope retention and transport in metals a macroscopic rate equation (MRE) modelling is typically used. MRE models are used to obtain relevant parameters for processes in the metal like trapping and diffusion [8, 9, 10, 11, 12]. The model couples both diffusion and trapping of hydrogen isotopes inside the bulk of the material. In our case we have used the TESSIM code [8]. The microscopic energy parameters included in the rate equations are obtained either from benchmark experiments e.g. 
[13], or by density functional theory (DFT) [14]. These parameters are defined by the interaction potential between a deuterium ion or atom and the material during particle impact onto the surface and while traveling through the bulk of the material. For a clear representation the interaction between hydrogen and a metal is presented in Fig. 1, using the potential energy curves for the interaction of hydrogen with the metal surface. Two potentials are shown as a function of distance from the surface. The blue line applies to hydrogen atoms interacting with the metal while the red line corresponds to the interaction of a molecule with the surface. Such one-dimensional diagrams show only the cross-sections through a multi-dimensional energy surface at specific inter-atomic distances.

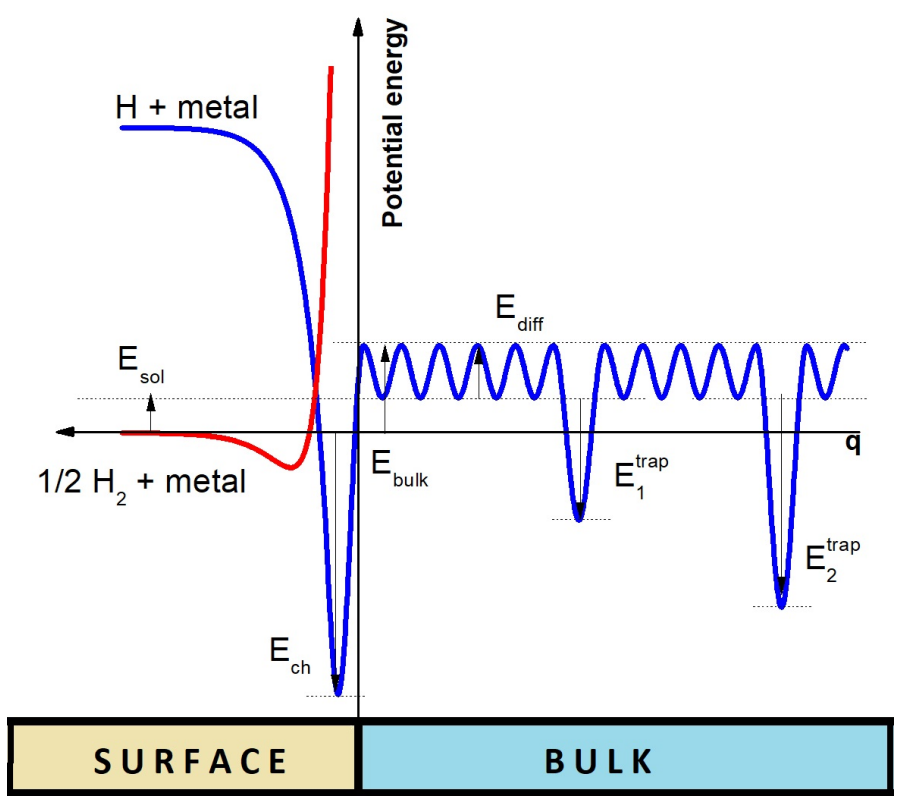

Figure 1: The potential that characterizes the interaction between an impacting hydrogen isotope atom (blue line) or a molecule (red line) with the surface and bulk of a metal. $q$ represents the reaction coordinate.

When a hydrogen molecule approaches the surface it needs to dissociate before it can interact strongly with the surface. The energy of a molecule far away from the surface is defined as zero energy as shown in the potential in Fig. 1 1 while atoms compared to molecules have more energy as they posses half of the dissociation energy. In principle one could think that atoms can penetrate directly into the bulk as their energy is higher than the potential barrier of the surface $E_{\text {bulk }}$. However, in our recent study [15] we show that direct penetration does not explain the experimental results and that atoms are trapped in chemisorption sites with a high probability. Their excessive energy is spent in electron-hole interactions as they diffuse on the surface. As the coverage of the surface increases as more D atoms get trapped at the surface, some of the excessive energy can also be spent for such a hot atom to penetrate into the bulk.

In summary, as a general rule of thumb, hydrogen atoms and ions with energies smaller than about $1 \mathrm{eV}$ get chemisorbed at the chemisorption site with energy $E_{c h}$, while $\mathrm{HI}$ ions or atoms with energies larger than $1 \mathrm{eV}$ they become directly implanted into the bulk [16]. 
After a HI ion or atom enters the bulk it diffuses through tetrahedral interstitial sites (TIS) with an activation energy for diffusion $E_{\text {diff }}$. The energy attributed to diffusion and the potential barrier for a chemisorbed atom to enter the bulk define the solution energy $E_{\text {sol }}=E_{\text {bulk }}-E_{\text {diff }}$. In case when defects are present in the material they are represented as different trap types $i$ with a deeper minimum with energy $E_{i}^{\text {trap }}$.

The model takes into account that there are two specific regimes to the interaction. The interaction between the incoming particle with the surface and the interaction of the hydrogen isotope atom with the bulk of the material. Equations that apply for the bulk are [8]:

$$
\begin{aligned}
\frac{\partial c^{s o l}(x, t)}{\partial t} & =D(T(t)) \frac{\partial^{2} c^{s o l}(x, t)}{\partial x^{2}}+S(x, t)-\sum_{i} \frac{\partial c_{i}^{\text {trap }}(x, t)}{\partial t} \\
\frac{\partial c_{i}^{\text {trap }}(x, t)}{\partial t} & =\frac{D(T(t))}{a_{0}^{2}} c^{s o l}(x, t)\left[\eta_{i}(x, t)-c_{i}^{\text {trap }}(x, t)\right]-c_{i}^{\text {trap }}(x, t) \nu_{i} \exp \left(-\left(E_{i}^{\text {trap }}+E_{d i f f}\right) / k T(t)\right)
\end{aligned}
$$

They govern the behavior of the deuterium atomic fraction $c_{i}^{\text {trap }}$ that is trapped in defect type $i$ and the solute deuterium atomic fraction $c^{s o l}$ that diffuses through TIS sites. As stated deuterium ions with energies higher than $1 \mathrm{eV}$ can penetrate directly into the bulk. This corresponds to the inclusion of $S(x, t)$ in equation 1 . In our case where the energy of deuterium atoms is much smaller than $1 \mathrm{eV}$ we can safely assume that $S(x, t)=0$. This means equation 1 is the standard diffusion equation with the addition of the possibility of a solute deuterium getting trapped in any of the various trap types in the material. Equation 2 describes the trapping and de-trapping of deuterium atoms in various defects. The diffusion coefficient used here is the one measured by Frauenfelder [17] where $D_{0}=4.1 \times 10^{-7} \mathrm{~m}^{-2} \mathrm{~s}^{-1}$ and $E_{\text {diff }}=0.39 \mathrm{eV}$. Because Frauenfelder measured these parameters for hydrogen, $D_{0}$ has to be divided by $\sqrt{2}$ to account for deuterium having twice the mass of hydrogen. To complete this set of equations we must also include the boundary conditions.

It is easily discernible that the surface acts as the boundary condition for the bulk. When ions are implanted deep into the bulk of the material we assume that the diffusion to the surface is the rate-limiting step, because the time-scales of diffusion from deep in the bulk to the surface are much larger than the time-scale of D desorption from the surface. This means we assume that a deuterium atom that comes to the surface of the material from the bulk desorbs immediately, as is usually assumed in modeling [18]. Meanwhile, if the surface is exposed to deuterium atoms, we have to include various fluxes that go to and from the surface and the atomic fraction of deuterium atoms $c_{A j}$ that are adsorbed in chemisorption sites on the surface. This is the so-called surface model that was described in detail in [13, 15].

The dominant fluxes to the surface are the flux of incoming particles that stick to the surface $\Gamma_{j}^{a d s}$ and atoms hopping from the bulk to the surface $\Gamma_{j}^{\text {surf }}$. Fluxes from the surface are due to Langmuir-Hinshelwood $\Gamma_{j}^{L H}$, Eley-Rideal recombination $\Gamma_{j}^{E R}$ and also atoms hopping from the surface into the bulk $\Gamma_{j}^{b u l k}$ 


$$
\begin{aligned}
\Gamma_{j}^{a d s} & =\frac{\Gamma_{0}}{\eta^{\text {surf }}}(1-R)\left(\eta_{j}^{\text {surf }}-c_{A j}(t)\right) \\
\Gamma_{j}^{E R} & =\Gamma_{0} \sigma_{E R} \delta_{W} c_{A j}(t) \\
\Gamma_{j}^{L H} & =2 k_{L H}\left(\delta_{W} c_{A j}(t)\right)^{2} \exp \left(-2 E_{c h} / k_{B} T\right) \\
\Gamma_{j}^{b u l k} & =\nu_{j} \delta_{W} c_{A j}(t) \exp \left(-\left(E_{c h}+E_{b u l k}\right) / k_{B} T\right) \\
\Gamma_{j}^{\text {surf }} & =\frac{D(T(t))}{a_{0}^{2} \eta^{\text {surf }}} \delta_{W} c^{\text {sol }}(0, t)\left(\eta_{j}^{\text {surf }}-c_{A j}(t)\right)
\end{aligned}
$$

$\Gamma_{0}$ is the flux of impinging deuterium particles. Here $(1-R)=0.15[19]$ is the sticking coefficient that describes the probability of a deuterium atom getting trapped in a free adsorption site. $\eta_{j}^{\text {surf }}$ is the atomic fraction of adsorption sites. $\sigma_{E R}$ is the Eley-Rideal cross section that describes the probability that an impinging deuterium atom abstracts an atom from the surface so they both leave it as a molecule. $k_{L H}=0.07 \mathrm{~cm}^{2} / \mathrm{s}$ is the coefficient for Langmuir-Hinshelwood recombination, where two adsorbed atoms recombine on the surface and leave as molecule [20]. $\delta_{W}=1.6 \times 10^{19} \mathrm{~m}^{-2}$ is the surface density of tungsten. The energies used in the formulae are defined in Fig. 1.

By including these fluxes we can write an equation that describes the atomic fraction of chemisorbed deuterium atoms $c_{A j}$ :

$$
\delta_{W} \frac{\partial c_{A j}(t)}{\partial t}=\Gamma_{j}^{a d s}+\Gamma_{j}^{s u r f}-\left(\Gamma_{j}^{E R}+\Gamma_{j}^{L H}+\Gamma_{j}^{b u l k}\right)
$$

and the equation that acts as the boundary condition for the D bulk atomic fraction:

$$
-D(T(t)) \rho_{W} \frac{\partial c^{s o l}(0, t)}{\partial x}=\sum_{j}\left(\Gamma_{j}^{b u l k}-\Gamma_{j}^{s u r f}\right)
$$

\section{Experiment}

For the purpose of this experiment we used three samples with different grain sizes named hot-rolled tungsten (HR-W), recrystallized tungsten (R-W), ITERGrade tungsten (IG-W) and a single crystal sample (SC-W) with surface orientation $<100>$.

\subsection{Samples}

Samples HR-W and R-W are hot rolled tungsten samples with dimensions $12 \times 15$ $\mathrm{mm}^{2}$ and thickness $0.8 \mathrm{~mm}$ originating from the same manufacturing batch produced by Plansee SE. The grains are oriented along the surface because of hot rolling. Sample IG-W was cut from a forged rod produced by Plansee, AG, such that its grains are oriented perpendicular to the surface. Sample SC-W is a single crystal. Its shape is circular with a diameter of $11 \mathrm{~mm}$.

All samples were prepared by electropolishing according to the procedure proposed by Manhard et al. [21]. Samples HR-W, SC-W and IG-W were heated in vacuum to a temperature of $1200 \mathrm{~K}$, which ensures that all retained hydrogen is desorbed and near surface defects introduced by the cutting and polishing of the 


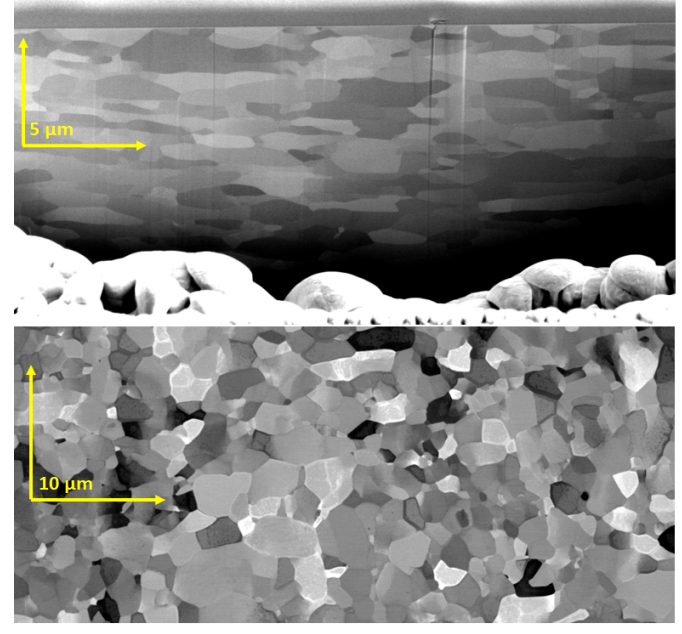

(a) HR - W

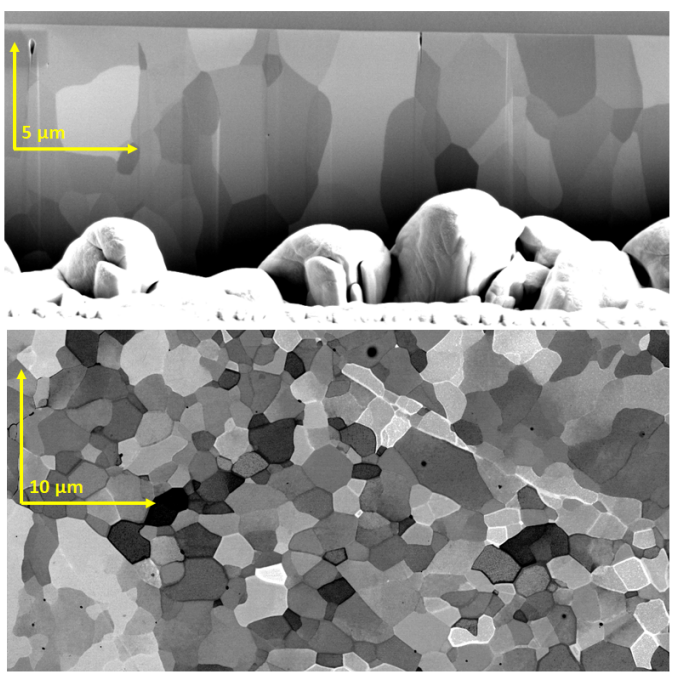

(c) IG - W

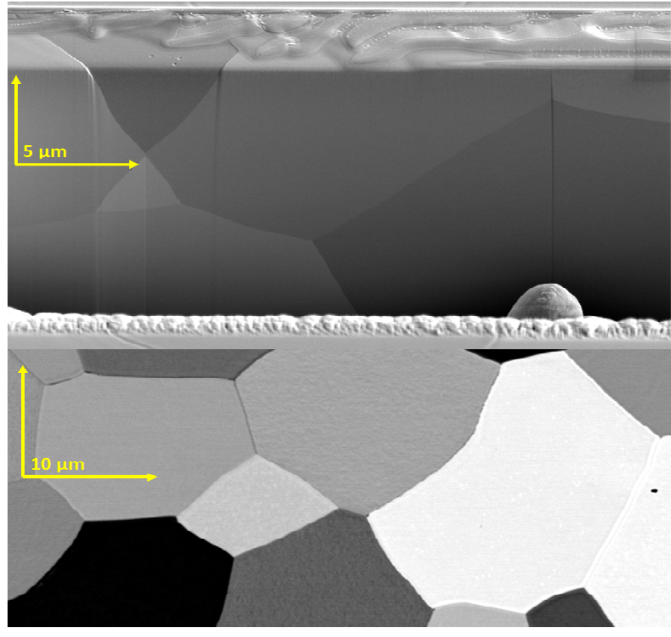

(b) $\mathbf{R}-\mathbf{W}$
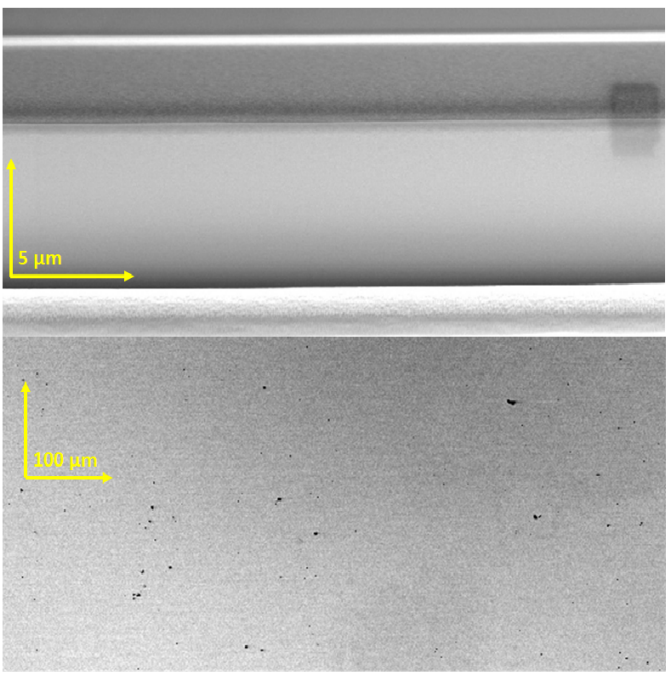

(d) SC - W

Figure 2: The results of an SEM scan of a FIB cut for all four samples, which was carried out at IPP, Garching. The top part shows the SEM scan of the cross section made by the FIB cut taken at 38 degrees. The bottom part of the SEM scan shows the top-down view of the surface of each sample.

samples are removed. Sample R-W was heated to $2000 \mathrm{~K}$ in an UHV oven which enlarges the grains relative to sample HR-W and reduces the grain boundary as well as dislocation density [22].

To increase deuterium sensitivity and retention the samples were self-damaged at room temperature with $20 \mathrm{MeV} \mathrm{W}^{6+}$ at the TOF beam line at the tandem laboratory of IPP, Garching, 23. Samples HR-W, R-W and SC-W where damaged with fluence $7.8 \times 10^{17} \mathrm{~m}^{-2}$ which produced a calculated damage dose equal to 0.25 dpa in the peak maximum. This was calculated with the program SRIM [24] where we used the quick damage calculation option which is based on the modified Kinchin Pease model by Norgett-Robinson-Torrens [25] using the W displacement energy of $90 \mathrm{eV}$ and the lattice binding energy $3 \mathrm{eV}$.

The sample IG-W was damaged with a fluence of $1.4 \times 10^{18} \mathrm{~m}^{-2}$ which produced damage with a dose of $0.45 \mathrm{dpa}$. The higher damage dose was not expected to 
influence the measurement, because it was shown [6, 26, 27] that the deuterium atomic fraction in self-damaged tungsten saturates at damage doses greater than the damage saturation value of tungsten fluence. As 't Hoen has shown [27] a safe estimate to where deuterium saturation occurs is with a $\mathrm{W}$ fluence in the range of $2-5 \times 10^{17} \mathrm{~W} / \mathrm{m}^{2}$ for damaging at room temperature. Because in our case the $\mathrm{W}$ damaging fluences are much larger than the saturation $\mathrm{W}$ fluence we are positive that the saturation has occurred in both cases of damaging.

The grain structure of all four samples can be seen in Fig. 2. The top part of each figure shows Scanning Electron Microscope (SEM) pictures of an area of the samples cross section which was cut by a Focused Ion Beam (FIB). The cut was performed perpendicular to the surface and the SEM image is performed under an angle of 38 degrees. This allows us to see the surface and the bulk grain structure of the sample. The bottom part of each figure shows a top view of the surface grain structure of the samples. We can see that the samples have varying surface grain size. Samples IG-W and HR-W have very similar surface grain sizes but have drastically different grain structures in the bulk. The grains in IG-W are elongated preferentially perpendicular to the surface while in the case of HR-W the grains are elongated along the surface.

By knowing the combined area of the top-down SEM scan and the number of grains in the same scan we can get the average area of a grain for all samples. By assuming that grains have a square shape we can get the average size of grains on the surface for all samples. These are: $d_{H R-W}=1.4 \mu \mathrm{m}, d_{I G-W}=1.6 \mu \mathrm{m}$, $d_{R-W}=17.3 \mu \mathrm{m}$. Despite HR-W and IG-W having very similar grain sizes on the surface the dimension of their respective grains perpendicular to the surface is very much different. We also assume we can describe the sample SC-W as a sample with an infinite grain, therefore $d_{S C-W}=\infty$.

\subsection{Experimental set-up}

$\mathrm{D}$ atom loading and depth profiling was carried out at the Tandetron accelerator at Jožef Stefan Institute (JSI). The samples were exposed to a low energy deuterium atom beam from a hydrogen atom beam source (HABS) that was $73 \mathrm{~mm}$ away from the surface of the sample. The angle of impact on the surface was $51^{\circ}$. The driving pressure in the HABS source was $33.3 \mathrm{~Pa}$ which produced a maximum flux of $(4.1 \pm 0.3) \times 10^{18} \mathrm{D} / \mathrm{m}^{2} \mathrm{~s}$ on the sample surface. The flux was determined by measuring the erosion of a thin a-C:H film [28] as was explained in a previous paper [29]. The tungsten capillary of the HABS source was heated to $T_{H A B S}=2080 \mathrm{~K}$ which produced deuterium atoms with energy $E=0.27 \mathrm{eV}$. The exposure took place in the INSIBA vacuum chamber where the samples were directly clamped to a boraelectric heating element. The temperature of the sample was set to $600 \mathrm{~K}$ and measured at all times by two shielded type $\mathrm{K}$ thermocouples, one pressed against the surface of the sample and one inside the heating element. The geometry of the set-up can be seen in Fig. 3.

Each sample was exposed for 70 hours. During the exposure we measured the transport of deuterium into each sample by monitoring the depth profile of D. For this we applied Nuclear Reaction Analysis (NRA) with a ${ }^{3} \mathrm{He}$ ion beam that impacted the sample perpendicularly on a $2 \mathrm{~mm}$ diameter area. The $D\left({ }^{3} \mathrm{He}, p\right) \alpha$ nuclear reaction was used for $\mathrm{D}$ detection [30]. The depth profile of $\mathrm{D}$ in the samples can 


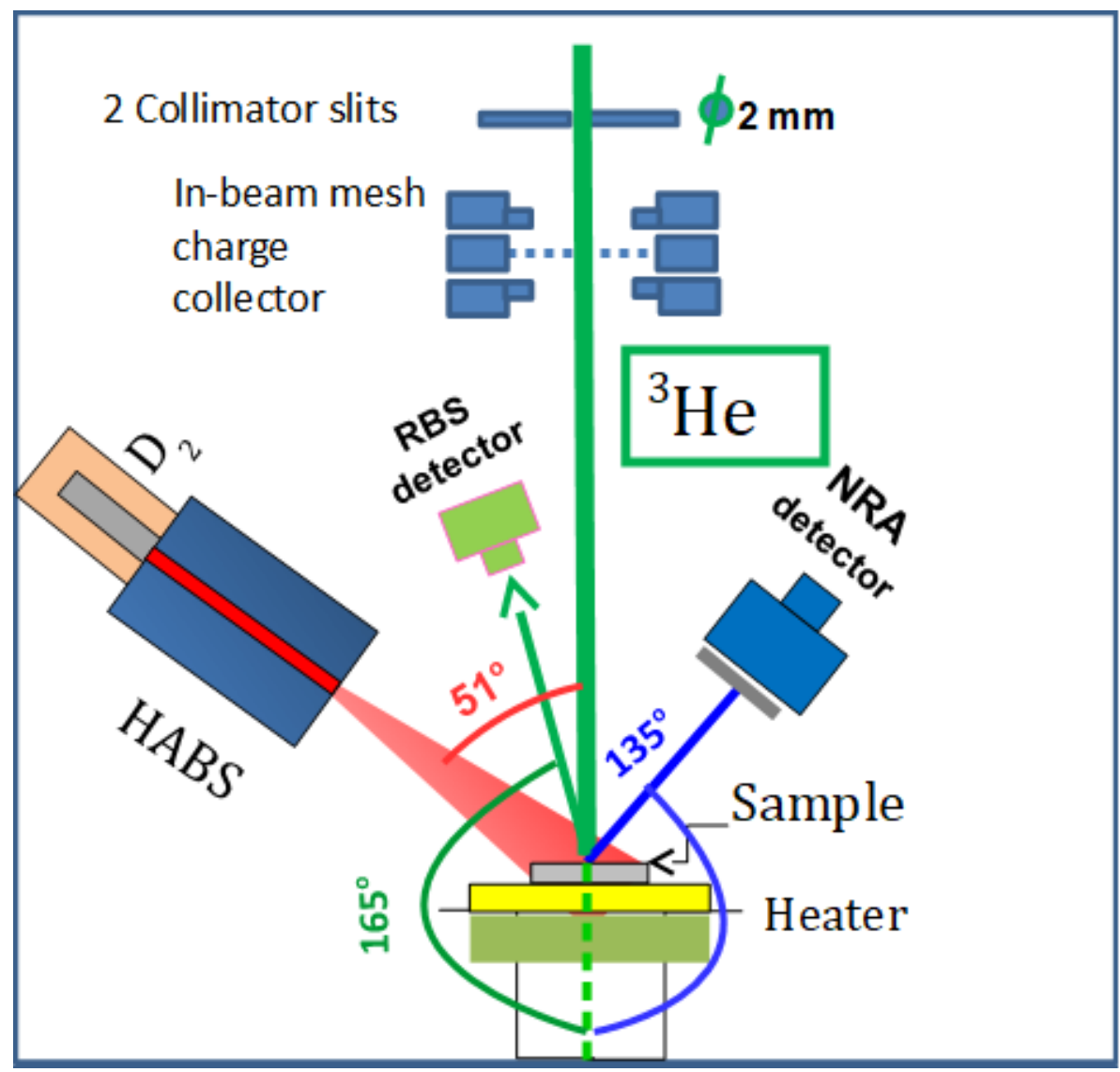

Figure 3: The geometry of the sample relative to the ${ }^{3} \mathrm{He}$ ion beam and the detectors that are used for studying of transport of deuterium into the samples.

be deduced by measuring with several ${ }^{3} \mathrm{He}$ ion beam energies and deconvoluting the proton energy spectra [31]. The energies of the ${ }^{3} \mathrm{He}$ ion beam were $0.7 \mathrm{MeV}, 1 \mathrm{MeV}$, 1.5 MeV, $2.5 \mathrm{MeV}, 3.3 \mathrm{MeV}$ and $4.2 \mathrm{MeV}$. At each measured energy the accumulated ion dose, that was measured by the in-beam mesh charge collector [32] with $77.4 \%$ transparency, was $4 \mu \mathrm{C}$. The total number of ${ }^{3} \mathrm{He}$ ions that hit the surface of the sample at each measured energy was $N_{H e}=8.56 \times 10^{13}$ for energies $0.7 \mathrm{MeV}, 1 \mathrm{MeV}$, $1.5 \mathrm{MeV}, 2.5 \mathrm{MeV}$ and $N_{H e}=4.28 \times 10^{13}$ for energies $3.3 \mathrm{MeV}$ and $4.2 \mathrm{MeV}$.

The proton spectrum generated in the reaction was measured by a Passivated Planar Silicon (PIPS) detector with a $1.5 \mathrm{~mm}$ thick depletion layer which was positioned at an angle of $135^{\circ}$ in relation to the impacting helium ion beam and captured a spatial angle of $26.7 \mathrm{msr}$. Any backscattered He ions were stopped by a $24 \mu \mathrm{m}$ thick Al foil. Another PIPS detector captured back-scattered particles for Rutherford backscattering (RBS) analysis. It was set up at $165^{\circ}$ relative to the He ion beam. In front of it was a $0.8 \mu \mathrm{m}$ thick $\mathrm{Al}$ foil that minimizes the detection of photons that are created by the HABS source. Such a set-up and the used set of energies allows a depth resolution of $0.25 \mu \mathrm{m}$ at the surface which steadily decreases to $1.1 \mu \mathrm{m}$ at a depth of $3 \mu \mathrm{m}$. The maximum depth of detection is about $7 \mu \mathrm{m}$.

After the exposure to D atoms a Thermal Desorption Spectroscopy measurement on each of the samples was carried out in a quartz tube of the TESS setup at IPP, Garching [33. The samples were heated from $300 \mathrm{~K}$ to $1000 \mathrm{~K}$ with a heating ramp of $T_{\text {ramp }}=3 \mathrm{~K} / \mathrm{min}$. Time traces of 16 preselected mass channels were recorded with a quadrupole mass spectrometer in ion counting mode, including $2 \mathrm{u} / \mathrm{q}, 3 \mathrm{u} / \mathrm{q}$, 
$4 \mathrm{u} / \mathrm{q}$ and $18 \mathrm{u} / \mathrm{q}, 19 \mathrm{u} / \mathrm{q}$ and $20 \mathrm{u} / \mathrm{q}$ for hydrogen and deuterium containing species as well as $12 \mathrm{u} / \mathrm{q}$ up to $16 \mathrm{u} / \mathrm{q}$ for hydrocarbons as well as $28 \mathrm{u} / \mathrm{q}, 32 \mathrm{u} / \mathrm{q}$ and $44 \mathrm{u} / \mathrm{q}$ for nitrogen, carbon monoxide, carbon dioxide and oxygen. Deuterium desorption was dominated by $\mathrm{D}_{2}$ desorption signals with minor amounts of HD. Desorption of heavier species was negligible. Absolute calibration for $\mathrm{D}_{2}$ and $\mathrm{HD}$ was performed as outlined in Ref. [33. By modeling the TDS spectra using the rate-equation model presented above we deduced information about the trap densities and their respective trapping energies.

\section{Experimental Results}

The deuterium depth profiles were measured at approximately the same exposure times for all samples after $3 \mathrm{~h}, 8 \mathrm{~h}, 24 \mathrm{~h}, 30 \mathrm{~h}, 48 \mathrm{~h}, 53 \mathrm{~h}$ and after the end of exposure (70h). The final exposure time approximates to an atom fluence of $1.0 \times 10^{24} \mathrm{D} / \mathrm{m}^{2}$. Deuterium profiles were derived by deconvoluting the NRA proton spectra from the $D\left({ }^{3} \mathrm{He}, p\right) \alpha$ with NRADC [31]. Depth profiles of deuterium after the exposure to individual exposure times for all four samples are shown in Fig. 4. We can see that the deuterium depth profile has a box like shape for all cases with a maximum concentration of about 0.4 at. $\%$ and also that with increasing the exposure time or fluence it extends deeper into the material. This means that D is slowly diffusing into our sample in all cases with longer exposure time. For all the samples the deuterium reaches a depth of $2 \mu \mathrm{m}$ which agrees well with the damaging range due to irradiation by $20 \mathrm{MeV} \mathrm{W}$ ions. With additional $\mathrm{D}$ atom exposure the maximum $\mathrm{D}$ atomic fraction in the damaged zone increases a bit more and the $\mathrm{D}$ atomic fraction in the undamaged area, beyond $2 \mu \mathrm{m}$, also increases. This increase in the undamaged zone means that $\mathrm{D}$ diffused beyond the self-damaged zone and started to decorate the natural defects.

The final maximum $\mathrm{D}$ atomic fraction in the damaged zone is similar for all samples being $c_{H R-W}^{\text {trap }}=(0.44 \pm 0.04)$ at. $\%, c_{I G-W}^{\text {trap }}=(0.37 \pm 0.02)$ at. $\%, c_{R-W}^{\text {trap }}=$ $(0.40 \pm 0.03)$ at. $\%$ and $c_{S C-W}^{\text {trap }}=(0.38 \pm 0.02)$ at. \%. The atomic fractions in the layer from $1.93-3.71 \mu \mathrm{m}$ area are: $c_{H R-W}^{\text {undam }}=(0.063 \pm 0.015)$ at. $\%, c_{R-W}^{\text {undam }}=(0.040 \pm$ $0.009)$ at. $\%, c_{I G-W}^{\text {undam }}=(0.060 \pm 0.013)$ at. $\%$ and $c_{S C-W}^{\text {undam }}=(0.012 \pm 0.003)$ at $\%$, while the atomic fractions in the layer from $3.71-7.23 \mu \mathrm{m}$ are: $c_{H R-W}^{\text {undam }}=(0.019 \pm 0.005)$ at. $\%, c_{I G-W}^{\text {undam }}=(0.020 \pm 0.007)$ at. $\%, c_{R-W}^{\text {undam }}=(0.015 \pm 0.004)$ at. $\%$, and $c_{S C-W}^{\text {undam }}=(0.012 \pm 0.003)$ at $\%$. When observing the behavior of $\mathrm{D}$ atomic fraction in the undamaged zone it appears as if a pattern is emerging with samples with larger grains having smaller D atomic fraction and vice-versa. Special care must be taken before coming to such a conclusion. We suspect that the retention of $\mathrm{D}$ in the layer from 1.93 - $3.71 \mu \mathrm{m}$ still belongs into the damaged zone, because the created damage actually reaches up to about $2.3 \mu \mathrm{m}$. But because the NRA measurement cannot differentiate a $0.4 \mu \mathrm{m}$ layer at a depth of $2 \mu$ the fitting procedure offers a much thicker layer with a lower $\mathrm{D}$ atomic fraction. Therefore, to truly see an indication in trapping in grain boundaries one must observe the behavior of $\mathrm{D}$ in the deepest most layer ranging from $3.71-7.23 \mu \mathrm{m}$. There is an indication that there is a systematic difference in D atomic fraction, HR-W and IG-W having the largest, $\mathrm{R}-\mathrm{W}$ a smaller and SC-W having the smallest $\mathrm{D}$ atomic fraction. This difference can also be explained by the fact that samples R-W and SC-W were heated to a very high temperature of $2000 \mathrm{~K}$ during sample preparation. This heating not only 


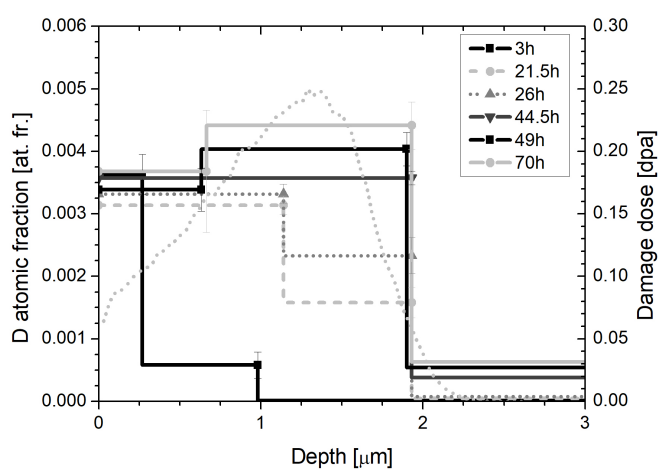

(a) HR - W

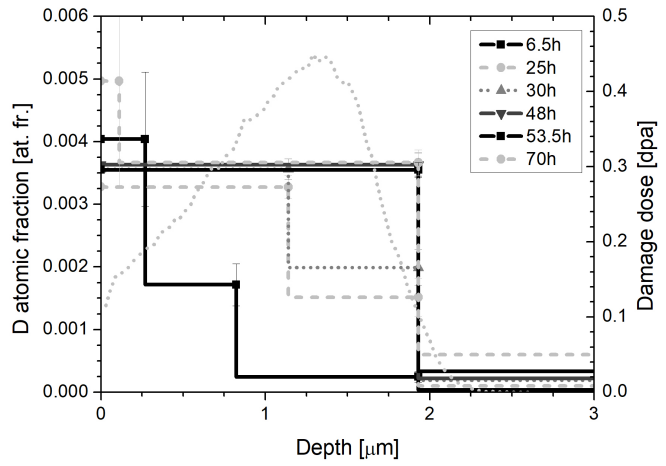

(c) IG - W

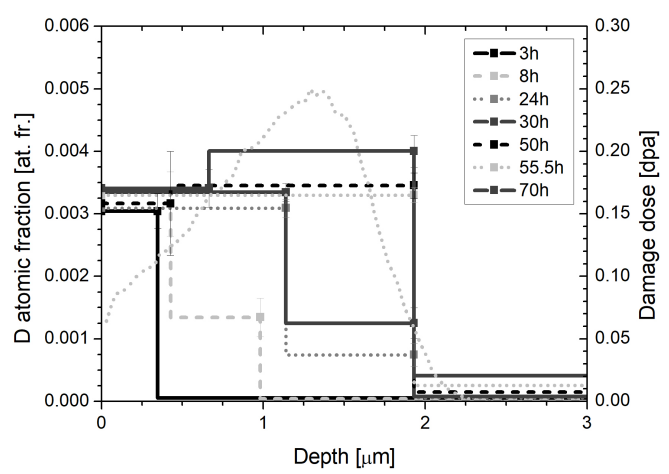

(b) $\mathrm{R}-\mathrm{W}$

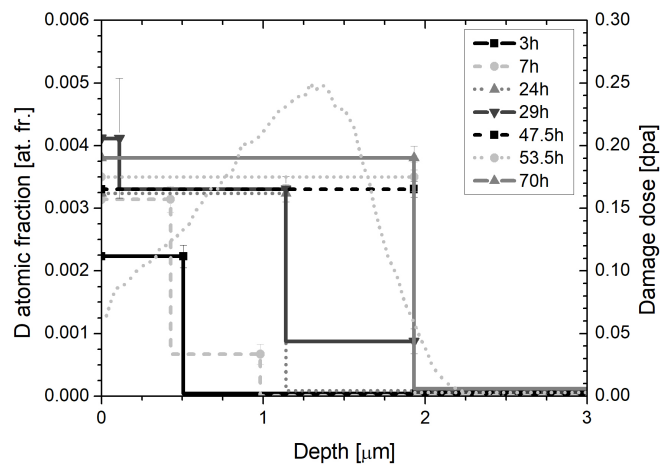

(d) $\mathrm{SC}-\mathrm{W}$

Figure 4: Depth profiles for deuterium of all four samples exposed at $600 \mathrm{~K}$ to a flux of $(4.1 \pm 0.3) \times 10^{18} \mathrm{D} / \mathrm{m}^{2} \mathrm{~s}$. The damage dose depth profile is drawn in gray.

enlarges the grains which was needed in the case for R-W, but also decreases the amount of intrinsic defects present in the samples. Because HR-W and IG-W were not heated to such high temperatures, they still contain a relatively large density of intrinsic defects. Which of the two explanations dominate cannot be inferred.

The errors in maximum concentrations stated here and in Fig. 4 come from the fitting process in the program NRADC which takes into account the counting errors of proton spectra acquisition and the error of ${ }^{3} \mathrm{He}$ ion dose measurement, used for calibration.

The total retained amount of deuterium in the samples is obtained by summing up the area under the NRA depth profiles. The result can be seen in Fig, $5 \mathrm{a}$ as a function of exposure time. The error bars in Fig. $5 \mathrm{a}$ are calculated as the sum errors in D retention in specific layers in the depth profile seen in Fig. 4. As can be observed the uptake is very similar for all samples at the 4 hours of exposure. The penetration depth is not more than $250 \mathrm{~nm}$, as we can see in Fig. 4. The penetration depth after $30 \mathrm{~h}$ when the total deuterium amounts start to deviate from each other is $1 \mu \mathrm{m}$. There the single crystal and the $\mathrm{R}-\mathrm{W}$ show smaller retained amount as compared to IG-W and HR-W. After $70 \mathrm{~h}$ the total amount of retained D differ by about $20 \%$ if we compare the samples HR-W and SC-W. We can conclude from this that samples with smaller grains (HR-W and IG-W) show an increased rate of deuterium uptake compared to samples with larger grains (SC-W and R-W).

We can observe that in the case of the sample IG-W there is a fall in the retention of $\mathrm{D}$ in the last two data points at $53.5 \mathrm{~h}$ and $70 \mathrm{~h}$. This can be explained by the fact that the analyzing spot on the sample was moved by $2 \mathrm{~mm}$ for the last 20 


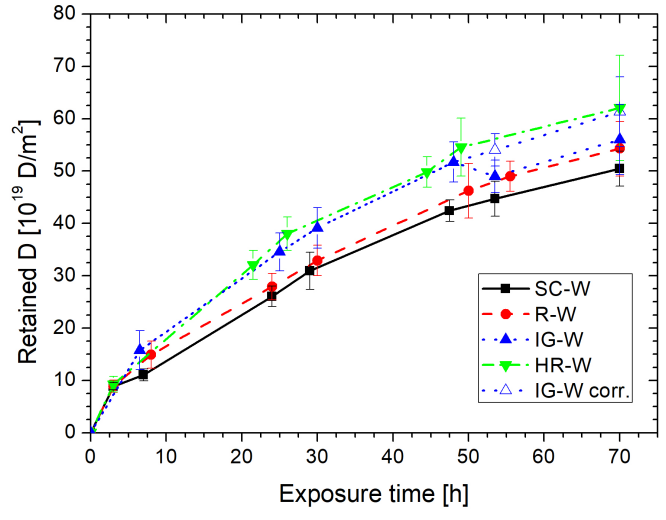

(a)

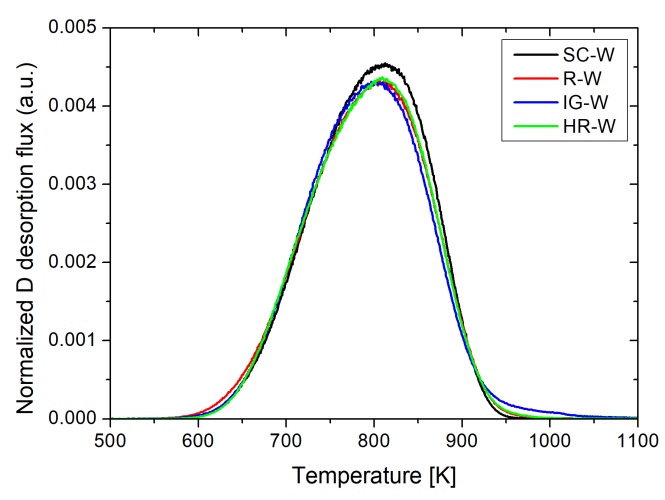

(b)

Figure 5: (a) Retention of deuterium in the damaged layer as a function of exposure time for all four samples as derived from NRA measurements. For IG-W two sets of data are plotted. Full blue triangles represent the experimental data as derived from the integration of the depth profiles, while the empty blue triangles are experimental values corrected because of the lower flux in the last two data points at $53.5 \mathrm{~h}$ and 70 h. (b) TDS spectra for all four samples were normalized to their individual integrals.

hours to see a possible effect of the analyzing ${ }^{3} \mathrm{He}$ beam on $\mathrm{D}$ retention. For the first 50 hours of the experiment the flux of atoms at this new analyzing spot was $(3.1 \pm 0.3) \times 10^{18} \mathrm{D} / \mathrm{m}^{2} \mathrm{~s}$ which was seen as a negligible effect on the $\mathrm{D}$ retention at the time. As we discovered later through simulation the lower atomic flux decreases the retention by about $10 \%$. Therefore, for ease of comparison with other samples the relevant data points at $53.5 \mathrm{~h}$ and $70 \mathrm{~h}$ were corrected by adding $10 \%$ to the experimental retention. In Fig. 5 a the original experimental data points are plotted with empty blue triangles, while the corrected values are plotted with full blue triangles. The effect of the lower atomic flux and how the lowered retention compares with the data points at $53.5 \mathrm{~h}$ and $70 \mathrm{~h}$ can be seen in Fig. 6 where a simulation of the experiment is shown.

We can also see that D depth profiles show some similarities with the calculated damage profile seen in Fig. 4 where they are drawn as a grey line. Mainly samples HR-W and R-W show a matching depth of the damaging peak and the peak of $\mathrm{D}$ retention. From the time evolution of the depth profiles one can observe that first the entire damaged layer is saturated, only then the damage peak starts to form. Samples IG-W and SC-W do not show this same behavior of the D fraction somewhat following the damage profile. In sample $\mathrm{SC}-\mathrm{W}$ this is due to a slower uptake of D into the sample compared to the other samples therefore the peak of the damage zone has not yet been fully saturated as it has in the case in samples R-W and HR-W. One would expect the D atomic fraction depth profile to start showing the damage peak feature if the $\mathrm{D}$ fluence would be larger. One would also expect the damage peak to be saturated in the case of IG-W because its grain structure is similar to that of HR-W. The absence of this peak can be explained by the fact that in the case of the samples HR-W, R-W and SC-W which were damaged to 0.25 dpa, true saturation of displacement damage occurs only near the position of the damage peak, while the area near the surface is only near saturation. Meanwhile, because IG-W was damaged to almost twice this amount, specifically to $0.45 \mathrm{dpa}$, true saturation occurs in the entire damage region. 
In Fig, $5 \mathrm{~b}$ the $\mathrm{D}$ desorption flux as a function of sample temperature for all four samples is shown. The D desorption flux shown has been normalized to the respective total retained $D$ amount for each sample which was determined as the integral of the desorption flux. Direct comparison of the non-normalized desorption spectra is impossible due to difficulties with determining the exact areas of damaging and D atomic beam exposure. From the normalized D desorption flux for all samples we can observe that they show a very similar desorption signal consisting of one peak with a maximum at about $800 \mathrm{~K}$.

\section{Simulation}

To successfully simulate the results of the experiment we need some initial input to reduce the number of free parameters of the simulation. More specifically we need to extract information on surface adsorption sites from previous experiments as our experiment was limited to only one exposure temperature.

In a previous experiment conducted by Založnik et al. [15] also in the INSIBA vacuum chamber, the influence of sample temperature on deuterium transport and retention in self-damaged tungsten was studied. The samples in the experiment of Založnik were recrystallized tungsten originating from the same manufacturing batch and were prepared and damaged in the same way as our R-W sample. The selfdamaged tungsten samples were exposed to a low-energy deuterium atom beam with a flux of $4.2 \times 10^{18} \mathrm{D} / \mathrm{m}^{2} \mathrm{~s}$ for 121 hours. The samples were exposed at temperatures equaling $450 \mathrm{~K}, 500 \mathrm{~K}, 550 \mathrm{~K}$ and $600 \mathrm{~K}$. During the exposure in situ NRA was used to follow the time evolution of the retained D amount. After the exposure a TDS analysis was performed. To model the results of the experiment the same model in the TESSIM code was used as in our case. The modeling of the experiment yielded the energies and densities of defects in the bulk and the energies and densities of surface adsorption sites.

Založnik et al. identified two chemisorbtion sites one with energy $E_{1}^{c h}=(0.68 \pm$ $0.02) \mathrm{eV}$ and density $\eta_{1}^{c h}=(0.51 \pm 0.05) \times 10^{19} \mathrm{D} / \mathrm{m}^{2}$ and the other with energy $E_{2}^{c h}=$ $(0.71 \pm 0.02) \mathrm{eV}$ and density $\eta_{2}^{c h}=(1.1 \pm 0.1) \times 10^{19} \mathrm{D} / \mathrm{m}^{2}$. We use this information on surface adsorption sites as input parameters for our simulation. Because of the similar energies of both sites, we combine them into one chemisorption site with energy $E^{c h}=0.7 \mathrm{eV}$ and density $\eta^{c h}=1.61 \times 10^{19} \mathrm{D} / \mathrm{m}^{2}$ for the purpose of our experiment.

Založnik also reports two bulk trapping sites with energies $1.87 \mathrm{eV}$ and $1.6 \mathrm{eV}$. Because of the high exposure temperature in our experiment $(600 \mathrm{~K})$ only the high energy trap is active and for this reason also only one desorption peak is observed in Fig. 5b. By simulating our TDS spectra we deduced the energy of the trap corresponding to the single peak being $E^{\text {trap }}=1.82 \mathrm{eV}$. This is in good agreement with the value of $1.87 \mathrm{eV}$ determined by Zaloznik et al. and with experiments that have studied similar samples [34, 35].

Beside the atomic fraction of D retained in the high energy trap in individual samples which we will deduce from the maximum retained deuterium atomic fraction in the experimental depth profiles we now have all the parameters needed to successfully simulate our experiments. All of the parameters are listed in Tab. 1.

We assume that all samples have equal properties and that the only difference between them is the size of their respective grains. We have decided that a parameter 
Table 1: Table of all parameters needed to successfully simulate our experiment.

\begin{tabular}{llll}
\hline$D_{0}$ & Diffusion constant for deuterium in tungsten & $2.9 \times 10^{-7} \mathrm{~m}^{2} \mathrm{~s}^{-1}$ & {$[17]$} \\
\hline$E_{d i f f}$ & Energy of diffusion & $0.39 \mathrm{eV}$ & {$[17]$} \\
\hline$\rho_{W}$ & Density of tungsten & $6.2 \times 10^{28} \mathrm{~m}^{-3}$ & \\
\hline$a_{0}$ & Lattice constant of tungsten $\rho_{w}^{-1 / 3}$ & $0.25 \mathrm{~nm}$ & \\
\hline$\Gamma_{0}$ & Flux of deuterium atoms with energy $0.27 \mathrm{eV}$ & $4.1 \times 10^{18} \mathrm{~m}^{-2} \mathrm{~s}^{-1}$ \\
\hline$T$ & Exposure temperature & $600 \mathrm{~K}$ & \\
\hline$\sigma_{E R}$ & Cross section for Eley-Rideal recombination & $10^{-21} \mathrm{~m}^{2}$ & {$[29]$} \\
\hline$\delta_{W}$ & Surface density of tungsten $\rho_{w}^{2 / 3}$ & $1.6 \times 10^{19} \mathrm{~m}^{-2}$ & \\
\hline$k_{L H}$ & Coefficient of Langmuir-Hinshelwood recombination & $0.07 \mathrm{~cm}^{2} \mathrm{~s}^{-1}$ & {$[20]$} \\
\hline$R$ & Reflection coefficient for deuterium atoms on tungsten & 0.85 & {$[19]$} \\
\hline$\eta^{\text {ch }}$ & Density of chemisorption site & $1.61 \times 10^{19} \mathrm{D} / \mathrm{m}^{2}$ & {$[15]$} \\
\hline$E^{\text {ch }}$ & Energy of chemisorption site & $0.7 \mathrm{eV}$ & {$[15]$} \\
\hline$E^{\text {trap }}$ & Energy of the only relevant trap & $1.87 \mathrm{eV}$ & {$[15]$} \\
\hline$\nu^{\text {trap }}$ & Frequency of D attempting to escape the trap & $10^{13} \mathrm{~s}{ }^{-1}$ & {$[15]$} \\
\hline
\end{tabular}

of the potential shown in Fig. 1 that could be influenced by the size of the grains is the effective height of the potential barrier for atoms to migrate from the surface into the bulk. Namely, atoms that are adsorbed on the surface have to migrate over a high energy barrier which is according to literature $E_{c h}+E_{b u l k}=1.5 \mathrm{eV}[13]$ at similar exposure conditions. However, when grains are present some fraction of chemisorbed atoms can start their diffusion immediately on a grain boundary. Since these D atoms do not need to overcome the high potential barrier (or maybe it is much lower), the measured effective $E_{b u l k}^{e f f}$ is lower. This can be summed up in a simple equation:

$$
E_{\text {bulk }}^{\text {eff }}=\eta_{\text {surf }}^{G B} E_{\text {bulk }}^{\text {grain }}+\left(1-\eta_{\text {surf }}^{G B}\right) E_{\text {bulk }}^{S C}
$$

Here $\eta_{\text {surf }}^{G B}$ is the fraction of grain boundary sites on the surface, $E_{\text {bulk }}^{\text {grain }}$ is the potential barrier that a deuterium atom feels when it is chemisorbed on a grain boundary and $E_{b u l k}^{S C}$ is the intrinsic potential barrier of a surface without grain boundaries like the one of the single crystal sample - SC-W. Higher grain boundary density means higher fraction of atoms starting their way on a grain boundary, reducing the $E_{b u l k}^{e f f}$ even more. The atoms then diffuse along the grain boundary and present an additional source of atoms that can enter the grain and populate the traps. Even though the grain boundaries also act as a trap, due to their low trapping energy of $1 \mathrm{eV} \mathrm{[5]}$ an atom at $600 \mathrm{~K}$ can de-trap from the grain boundary and enter the grain. To try to validate this theory of grain boundary effect on $\mathrm{D}$ transport and retention we decided to only use $E_{b u l k}^{e f f}$ as a free parameter of the simulation.

Results for the retained amount of deuterium in the damaged layer were simulated using the TESSIM code which uses the model that was described earlier. The comparison between the best fit simulation and experiment can be seen in Fig. 6 . The best fit is achieved by minimizing the reduced $\chi^{2}$, which is defined as:

$$
\chi^{2}=\frac{1}{N-1} \sum_{i}\left(\frac{r\left(t_{i}\right)-r_{i}}{\delta r_{i}}\right)^{2}
$$

Here $N$ is the number of data points. $r\left(t_{i}\right)$ is the calculated retention at exposure 
time $t_{i}, r_{i}$ is the experimental retention at time $t_{i}$ and $\delta r_{i}$ is the experimental error of the retention at time $t_{i}$.

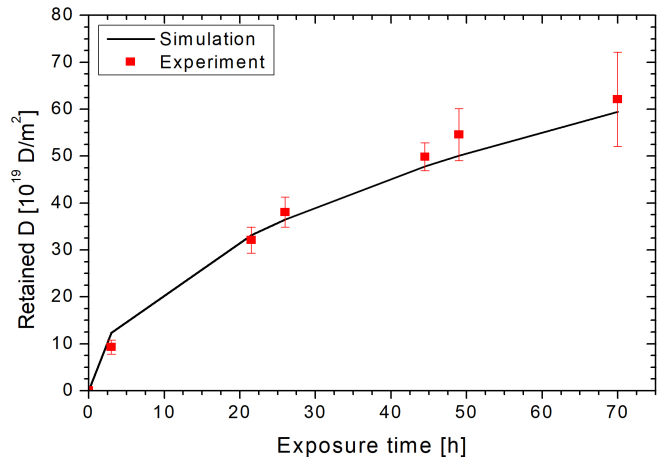

(a) HR-W

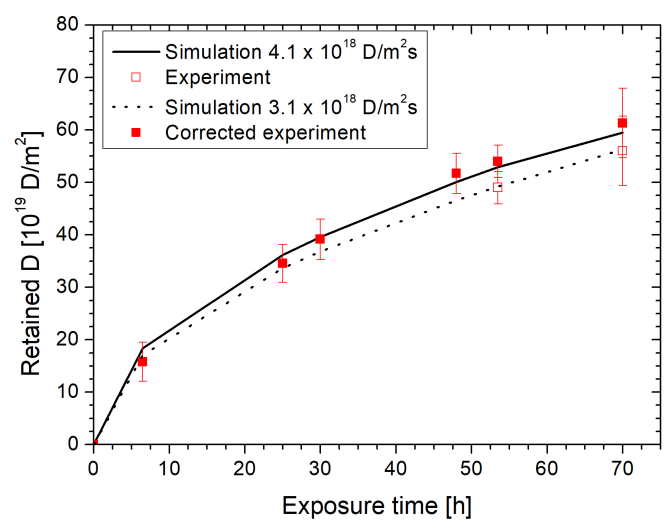

(c) IG-W

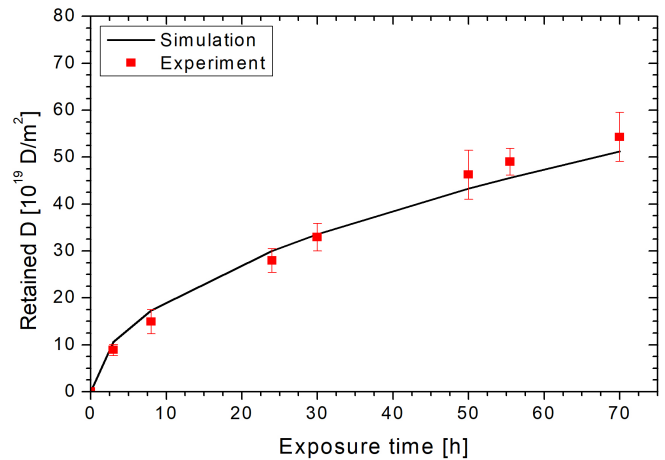

(b) R-W

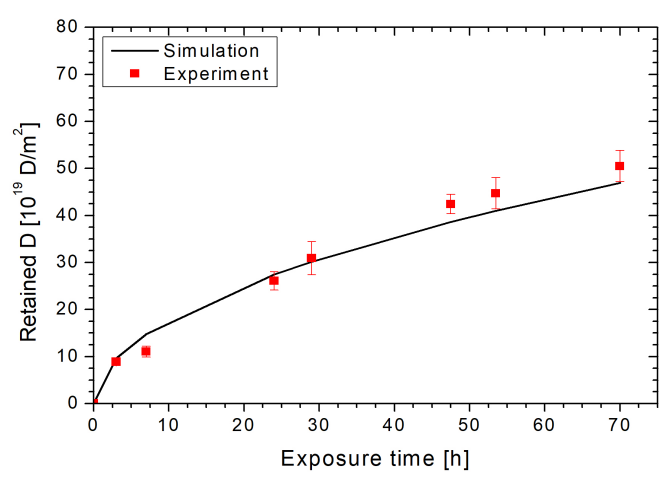

(d) SC-W

Figure 6: Comparison between the best fit simulation and experimental results for all four samples. In the case of IG - W two sets of data are compared. The dotted line represents the simulation of the experiment with flux $3.1 \times 10^{18} \mathrm{D} / \mathrm{m}^{2}$ s while the full line is the simulation of the experiment with flux $4.1 \times 10^{18} \mathrm{D} / \mathrm{m}^{2} \mathrm{~s}$. Empty red squares show the original experimental data derived from the depth profiles, while the full red squares represent the data where the last two D retentions measured at $53.5 \mathrm{~h}$ and $70 \mathrm{~h}$ are increased by $10 \%$ to account for the lower flux for easier comparison with the other samples.

We could successfully simulate the dependence of D retention on exposure time by changing the parameter $E_{b u l k}^{e f f}$ without introducing any new trap type that would account for trapping in grains. This confirms our assumption that at the exposure temperature of $600 \mathrm{~K}$ the presence of grain boundaries does not influence the maximal atomic fraction of retained $\mathrm{D}$ in self-damaged tungsten. The experimental results for all four samples could be reproduced very well by taking the same trap fraction of $\eta=0.42$ at. \% for all samples which is in accordance with the fact that all samples were damaged above saturation. The resulting best fit of the effective height of the potential barrier $E_{b u l k}^{e f f}$ and its respective reduced $\chi^{2}$ are stated in Tab. 2. The error of the fitting parameter $E_{b u l k}^{e f f}$ was acquired by finding the value of $E_{b u l k}^{e f f}$ that enlarges the reduced $\chi^{2}$ by one. The surface grain boundary densities were calculated that the width of a grain boundary is equal to the lattice constant of $\mathrm{W}$ and that the grains are squares. This is of course only an approximation but it is useful to get some representative values for surface grain boundary densities in the 
Table 2: The table contains best fit value for the effective height of the potential barrier $E_{b u l k}^{e f f}$ and its respective reduced $\chi^{2}$. The table also contains the surface grain boundary densities $\eta_{\text {surf }}^{G B}$, which where calculated assuming square grain shapes and a grain boundary width of one lattice constant of $\mathrm{W}$.

\begin{tabular}{ccccc}
\hline Sample & SC-W & R-W & IG-W & HR-W \\
\hline$\eta_{\text {surf }}^{\text {GB }}[$ at. \%] & 0 & $2.9 \times 10^{-5}$ & $3.1 \times 10^{-4}$ & $3.6 \times 10^{-4}$ \\
$E_{\text {bulk }}^{\text {eff }}[\mathrm{eV}]$ & $(0.730 \pm 0.07)$ & $(0.720 \pm 0.07)$ & $(0.703 \pm 0.08)$ & $(0.704 \pm 0.06)$ \\
$\chi_{\text {reduced }}^{2}$ & 2.651 & 0.837 & 0.194 & 0.947
\end{tabular}

samples.

In the case of IG-W an additional simulation was performed where all fitting parameters were kept fixed and only the atomic D flux was changed to $3.1 \times 10^{18}$ $\mathrm{D} / \mathrm{m}^{2} \mathrm{~s}$. This was done to confirm that the lowered $\mathrm{D}$ retention at exposure times of $53.5 \mathrm{~h}$ and $70 \mathrm{~h}$ was caused by lowered $\mathrm{D}$ atom flux as we hypothesized in the experimental results section. Indeed the match between the dotted line, representing the additional simulation and the data points plotted with empty red squares is very good. This confirms our hypothesis and justifies the corrections done to the experimental D retention.

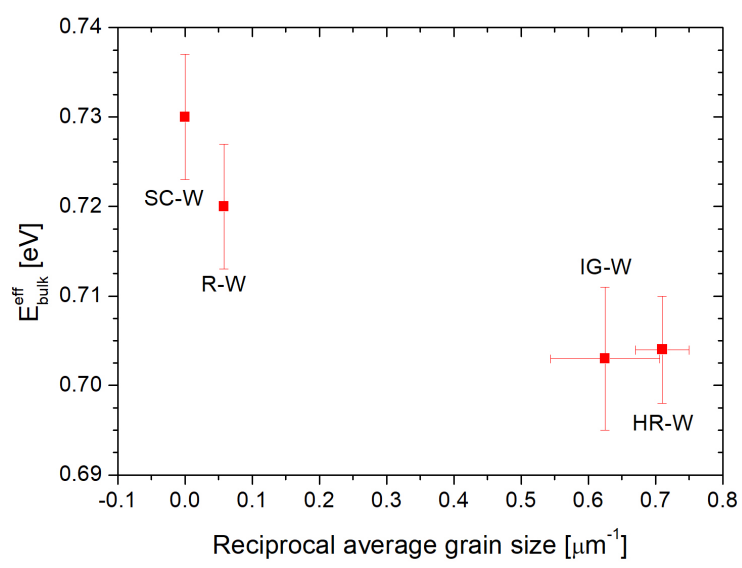

Figure 7: The effective height of the potential barrier for deuterium to enter the bulk as a function of inverse average grain size of the grains on the surface.

The plot of the effective height of the potential barrier as a function of inverse average grain size can be seen in Fig. 7. We can clearly see that the potential barrier depends on the inverse average grain size which is in fact directly proportional to the surface grain boundary density $\eta_{\text {surf }}^{G B}$. The effective height of the potential barrier for the sample R-W with the largest average grain size approaches the height of the effective barrier for the single crystal which has the highest effective height of the potential barrier and that can also be seen as the intrinsic potential barrier. Meanwhile the samples HR-W and IG-W with smaller average grain sizes have a smaller effective height of the potential barrier. 
By summing up the effective height of the potential barrier and the chemisorption energy we get approximately $E_{b u l k}^{e f f}+E_{c h}=1.4 \mathrm{eV}$ for all four samples. This is in good agreement from past results reported by Hodille et al. [13] but still lower than the results reported by Frauenfelder. This can be explained by the fact that Frauenfelder conducted his experiment at a much higher temperature of about a $1000 \mathrm{~K}$ where the surface D coverage was much lower than in our case where the experiment was done at $600 \mathrm{~K}$. Some DFT calculations [36] and also our own experimental results [37] indicate that the chemisorption energy of the surface depends on the D coverage, increasing with falling coverage. From our recent results [37] we see that indeed at higher exposure temperatures $E_{b u l k}^{e f f}+E_{c h}=1.9 \mathrm{eV}$ which is much closer to the value reported by Frauenfelder.

Taking the obtained parameters we also simulated the depth profiles and TDS spectra for individual samples. One example of such a simulation is shown for the case of R-W in Fig. 8. The obtained agreement of the simulation and the experimental results is good.

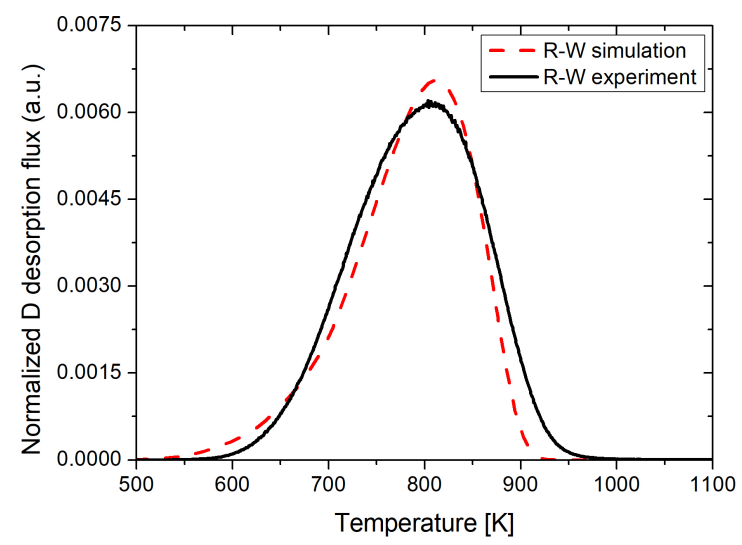

(a)

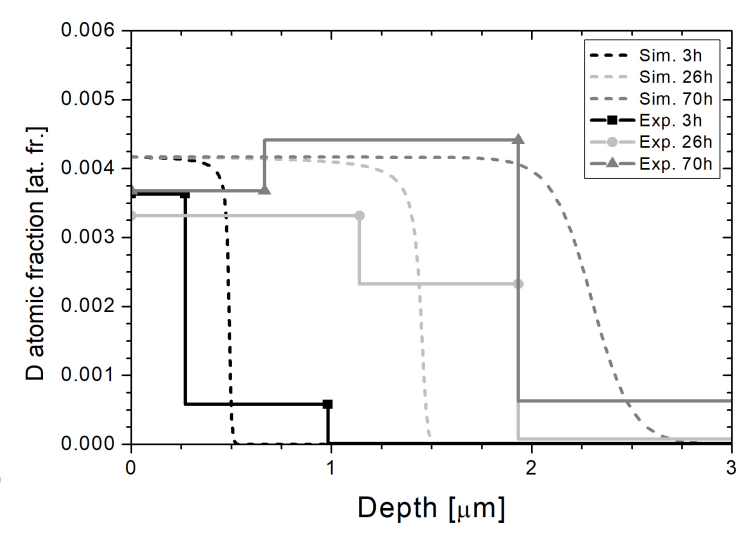

(b)

Figure 8: Comparison of the experimental result and the corresponding simulation for sample R-W. Experimental results are plotted with solid lines and simulations in dashed lines. (a) Comparison of the temperature dependence of the $\mathrm{D}$ desorption flux. The D desorption flux is again normalized to the total D retained amount. (b) Comparison of the D depth profile as a function of exposure time.

\section{Discussion and conclusions}

We have studied the effect of grain size on deuterium retention and transport in three different polycrystalline samples of tungsten and one tungsten single crystal with surface orientation $<100>$. The samples were self-damaged with $20 \mathrm{MeV} \mathrm{W}{ }^{6+}$ and later exposed to a deuterium atom beam at an exposure temperature of $600 \mathrm{~K}$ for 70 hours. During the exposure the depth profile of deuterium was monitored via ${ }^{3} \mathrm{He}$ NRA in situ. This allowed us to monitor any possible difference in deuterium transport that would occur because of different grain sizes of the samples. After the exposure a TDS measurement was carried out ex situ.

As we have shown in this paper the maximum deuterium atomic fraction in the bulk is not affected by different grain sizes in self-damaged tungsten at high temperatures of exposure. This starts to become clear when comparing the maximum 
deuterium atomic fraction retained in samples $\mathrm{HR}-\mathrm{W}$ with the smallest average grain size and the single crystal sample SC-W. Despite large differences in average grain sizes both samples show similar experimental maximum deuterium atomic fractions $c_{H R-W}^{\text {trap }}=(0.44 \pm 0.04)$ at. $\%$, and $c_{S C-W}^{\text {trap }}=(0.38 \pm 0.02)$ at. \% respectively. The simulation further strenghtens this argument as the smallest reduced $\chi^{2}$ was acquired for all of the samples when the same trap fraction of 0.42 at. \% was used to simulate the data despite each sample simulation being done independently. This has also been reported by Ogorodnikova and Sugiyama [38] who have shown that the only parameter that affects maximum deuterium atomic fraction in self-damaged $\mathrm{W}$ is the amount of damaging of the material and not characteristics like grain size, grain orientation, sample preparation and so on. Even though grain boundaries act as trapping sites for deuterium as reported by T. Oda [5], their contribution to the D retention can be neglected when conducting experiments at high enough exposure temperatures with self-damaged samples. This is further corroborated because there is only very little difference between the shape of the TDS spectra for all samples, as seen in Fig. 5b. On the contrary, some difference in D atomic fraction in the undamaged zone was observed, but its root cause remains unclear, as the difference can be explained by trapping of $\mathrm{D}$ in grain boundaries and also by trapping in intrinsic defects which remain in samples HR-W and IG-W, but were annealed away in $\mathrm{R}-\mathrm{W}$ and $\mathrm{SC}-\mathrm{W}$ during the re-crystallization process.

Despite grain boundaries not affecting the maximum deuterium atomic fraction retained in the material, we have shown that they influence the total retained amount of deuterium at a specific time of exposure as we can deduce from Fig. 5a. The difference of retained $\mathrm{D}$ after 70 hours of exposure between the sample with the largest grain SC-W and the sample with the smallest grain HR-W is about $20 \%$. This can be attributed solely to the difference in transport of $\mathrm{D}$ because of differences in grain sizes between the samples.

By simulating the transport of deuterium with the model in the TESSIM code we managed to describe the differences in the rate of deuterium uptake by changing only the effective height of the potential barrier for a deuterium atom hopping from the chemisorption site to the bulk of the material. We could successfully describe samples with smaller grain sizes with a smaller potential barrier while the potential barrier for samples with larger grain sizes neared its value for the single crystal sample. The differences in D retention as seen in Fig. 5a can be attributed exclusively to the fact that the samples have different potential barriers, as we were able to simulate the experimental results for all the samples with the same trap fraction.

We attribute this difference in the effective potential barriers to the fact that the samples have different surface grain boundary densities. Unfortunately we cannot reliably fit the dependence of $E_{b u l k}^{e f f}$ on inverse average grain size with Eq. 10 because of the large errors stemming from the simulation. The effective barrier $E_{b u l k}^{e f f}$ obtained from this experiment for $\mathrm{R}-\mathrm{W}$ is in good agreement with the previous studies [13, 15].

As we have seen the TDS spectra of all the samples are very similar while the differences in deuterium transport measured by D depth profiling are much clearer as seen in Fig. 5a. We do not see any difference in the TDS spectra since the analysis was performed after the entire depth of the damaged zone of the samples was almost completely decorated by deuterium. We should note that the difference in deuterium uptake for samples with different sized grains could also be possible to 
measure with the TDS technique alone if the damaged zone of the samples would not be fully saturated. Namely, one would expect to see different absolute values of the desorption peaks in the TDS spectra, because of faster or slower deuterium uptake and therefore larger or smaller retention respectively. But the effort to measure that would be much greater since for each fluence step a new sample would be needed.

We also expect that the shape of the TDS spectra would differ between the samples because D has penetrated to different depths in different samples for the same exposure time. This penetration range affects the shape of the TDS spectra. As was shown in [15] splitting of a TDS peak occurs if the damaged zone is not fully saturated, which could be easily misinterpreted since individual peaks are normally attributed to a specific detrapping energy.

Much has yet to be understood on the effect of grain boundaries on deuterium transport and retention. By conducting experiments on samples with grains in the scale of a few $100 \mathrm{~nm}$ we could possibly deduct a semi-empirical model for the behavior of the height of the potential barrier as a function of grain boundary surface density.

\section{Acknowledgement}

The authors of this article would like to thank G. Mattern who carried out the FIB analysis of our samples at IPP. We would also like to thank Mikhail Zibrov who allowed us to use one of his single-crystal samples for our experiment.

This work has been carried out within the framework of the EUROfusion Consortium and has received funding from the Euratom research and training programme 2014-2018 under grant agreement No 633053. Work was performed under WP PFC. The views and opinions expressed herein do not necessarily reflect those of the European Commission.

\section{Data availability}

The raw data required to reproduce these findings are available to download from https://data.mendeley.com/datasets/v7drzmjk6n/1. The processed data required to reproduce these findings are available to download from

https://data.mendeley.com/datasets/k2wshdjvvc/1.

\section{References}

[1] Y. Hatano et al., "Deuterium trapping at defects created with neutron and ion irradiations in tungsten," Nuclear Fusion, vol. 53, no. 7, p. 073006, 2013.

[2] T. Tanabe, "Review of hydrogen retention in tungsten," Physica Scripta, vol. T159, p. 014044, 2014.

[3] J. Marian, "Recent advances in modeling and simulation of the exposure and response of tungsten to fusion energy conditions," Nuclear Fusion, vol. 57, p. 092008, 2017. 
[4] G.-H. Lu, H.-B. Zhou, and C. S. Becquart, "A review of modelling and simulation of hydrogen behaviour in tungsten at different scales," Nuclear Fusion, vol. 54, p. 086001, 2014.

[5] T. Oda, "Thermodynamic mode for grain boundary effects on hydrogen solubility, diffusivity and permeability in poly-crystalline tungsten," Fusion Engineering and Design, vol. 112, pp. 102-116, 2016.

[6] O. V. Ogorodnikova and V. Gann, "Simulation of neutron-induced damage in tungsten by irradiation with energetic self-ions," Journal of Nuclear Materials, vol. 460, pp. 60-71, 2015.

[7] O. V. Ogorodnikova, "Effect of nanostructure on radiation tolerance and deuterium retention in tungsten," Journal of Applied Physics, vol. 122, no. 4, p. $044902,2017$.

[8] K. Schmid, V. Rieger, and A. Manhard, "Comparison of hydrogen retention in W and W/Ta alloys," Journal of Nuclear Materials, vol. 426, p. 247 - 253, 2012 .

[9] S. M. Myers, "Ion-beam studies of hydrogen-metal interactions," Journal of Nuclear Materials, vol. 165, pp. 9-64, 1989.

[10] M. A. Pick and K. Sonnenberg, "A model for atomic hydrogen-metal interactions - application to recycling, recombination and permeation," Journal of Nuclear Materials, vol. 131, pp. 208-220, 1985.

[11] E. A. Hodille et al., "Macroscopic rate equation modeling of trapping/detrapping of hydrogen isotopes in tungsten materials," Journal of $\mathrm{Nu}$ clear Materials, vol. 467, pp. 424-431, 2015.

[12] O. V. Ogorodnikova, "Fundamental aspects of deuterium retention in tungsten at high flux plasma exposure," Journal of Applied Physics, vol. 118, no. 7, p. $074902,2015$.

[13] E. A. Hodille et al., "Simulations of atomic deuterium exposure in self-damaged tungsten," Nuclear Fusion, vol. 57, no. 5, p. 056002, 2017.

[14] E. A. Hodille et al., "Retention and release of hydrogen isotopes in tungsten plasma-facing components: the role of grain boundaries and the native oxide layer from a joint experiment-simulation integrated approach," Nuclear Fusion, vol. 57, no. 7, p. 076019, 2017.

[15] A. Založnik, S. Markelj, T. Schwarz-Selinger, and K. Schimd, "Deuterium atom loading of self-damaged tungsten at different sample temperatures," Journal of Nuclear Materials, vol. 496, pp. 1 - 8, 2017.

[16] P. N. Maya, "Molecular dynamics studies of sticking and reflection of low-energy deuterium on single crystal tungsten," Journal of Nuclear Materials, vol. 480, p. $411,2016$.

[17] R. Frauenfelder, "Solution and diffusion of hydrogen in tungsten," The journal of vacuum science and technology, vol. 6, no. 3, pp. 388-397, 1968. 
[18] K. Schmid, J. Bauer, T. Schwarz-Selinger, and S. Markelj, "Recent progress in the understanding of $\mathrm{H}$ transport and trapping in W," Physica Scripta, vol. T170, p. 014037, 2017.

[19] O. V. Ogorodnikova, S. Markelj, and U. von Toussaint, "Interaction of atomic and low-energy deuterium with tungsten pre-irradiated with self-ions," Journal of Applied Physics, vol. 119, p. 054901, 2016.

[20] P. W. Tamm and L. D. Schmidt, "Binding states of hydrogen on tungsten," The Journal of Chemical Physics, vol. 54, pp. 4775-4787, 1971.

[21] A. Manhard, G. Matern, and M. Balden, "A step-by-step analysis of the polishing process for tungsten specimens," Praktische Metallographie, vol. 50, p. 6, 2013.

[22] A. Manhard, M. Balden, and S. Elgeti, "Quantitative microstructure and defect density analysis of polycrystalline tungsten reference samples after different heat treatments," Practical Metallography, vol. 52, no. 8, pp. 437-466, 2015.

[23] T. Schwarz-Selinger, "Deuterium retention in MeV self-implanted tungsten: Influence of damaging dose rate," Nuclear Materials and Energy, vol. 0, pp. 1-6, 2017.

[24] http://www.srim.org/.

[25] M. J. Norgett, M. T. Robinson, and I. M. Torrens, "A proposed method of calculating displacement dose rates," Nuclear Engineering and Design, vol. 33, p. 50, 1975.

[26] V. K. Alimov et al., "Deuterium retention in tungsten damaged with W ions to various damage levels," Journal of Nuclear Materials, vol. 441, pp. 280-285, 2013.

[27] M. H. J. 't Hoen et al., "Saturation of deuterium retention in self-damaged tungsten exposed to high-flux plasmas," Nuclear Fusion, vol. 52, p. 023008, 2012 .

[28] T. Schwarz-Selinger, A. von Keudell, and W. Jacob, "Novel method for absolute quantification of the flux and angular distribution of a radical source for atomic hydrogen," Journal of Vacuum Science \& Technology A: Vacuum, Surfaces, and Films, vol. 18, pp. 995-1001, 2000.

[29] S. Markelj et al., "In situ NRA study of hydrogen isotope exchange in self-ion damaged tungsten exposed to neutral atoms," Journal of Nuclear Materials, vol. 469, pp. 133-144, 2016.

[30] B. Wielunska et al., "Cross section data for the $\mathrm{d}(3 \mathrm{he}, \mathrm{p})$ 4he nuclear reaction from 0.25 to 6 mev," Nuclear Instruments and Methods in Physics Research B, vol. 371, pp. 41-45, 2015.

[31] K. Schmid and U. von Toussaint, "Statistically sound evaluation of trace element depth profiles by ion beam analysis," Nuclear Instruments and Methods in Physics Research Section B: Beam Interactions with Materials and Atoms, vol. 281, p. 64-71, 2012. 
[32] M. E. Bouanani et al., "Simple and accurate spectra normalization in ion beam analysis using a transmission mesh-based charge integration," Nuclear Instruments and Methods in Physics Research Section B: Beam Interactions with Materials and Atoms, vol. 243, pp. 392-396, 2006.

[33] P. Wang, W. Jacob, and L. Gao, "Comparing deuterium retention in tungsten films measured by temperature programmed desorption and nuclear reaction analysis," Nuclear Instruments and Methods in Physics Research Section B: Beam Interactions with Materials and Atoms, vol. 300, pp. 54-61, 2013.

[34] E. A. Hodille et al., "Study of hydrogen isotopes behavior in tungsten by a multi trapping macroscopic rate equation model," Physica Scripta, vol. T167, p. 014011, 2016.

[35] A. Založnik et al., "The influence of the annealing temperature on deuterium retention in self-damaged tungsten," Physica Scripta, vol. T167, p. 014031, 2016.

[36] Z. A. Piazza, M. Ajmalghan, Y. Ferro, and R. D. Kolasinski, "Saturation of tungsten surfaces with hydrogen: A density functional theory study complemented by low energy ion scattering and direct recoil spectroscopy data," Acta Materialia, vol. 145, pp. 388-398, 2018.

[37] E. Hodille, S. Markelj, T. Schwarz-Selinger, A. Zaloznik, M. Pecovnik, M. Kelemen, and C. Grisolia, "Stabilization of defects by the presence of hydrogen in tungsten: simultaneous w-ion damaging and d-atom exposure." submitted to Nuclear Fusion.

[38] O. V. Ogorodnikova and K. Sugiyama, "Effect of radiation-induced damage on deuterium retention in tungsten, tungsten coatings and Eurofer," Journal of Nuclear Materials, vol. 442, pp. 518-527, 2013. 